\title{
Is the exotic $X(5568)$ a bound state?
}

\author{
Xiaoyun Chen, Jialun Ping ${ }^{\mathrm{a}}$ \\ Department of Physics and Jiangsu Key Laboratory for Numerical Simulation of Large Scale Complex Systems, Nanjing Normal University, \\ Nanjing 210023, People's Republic of China
}

Received: 2 May 2016 / Accepted: 14 June 2016 / Published online: 24 June 2016

(C) The Author(s) 2016. This article is published with open access at Springerlink.com

\begin{abstract}
Stimulated by the recent observation of the exotic $X(5568)$ state by the D0 Collaboration, we study the fourquark system $u s \bar{b} \bar{d}$ with quantum numbers $J^{P}=0^{+}$in the framework of the chiral quark model. Two structures, diquark-antidiquark and meson-meson, with all possible color configurations are investigated by using the Gaussian expansion method. The results show that the energies of the tetraquark states with diquark-antiquark structure are too high to be candidates of $X(5568)$, and no molecular structure can be formed in our calculations. The calculation is also extended to the four-quark system $u s \bar{c} \bar{d}$ and the same results as that of $u s \bar{b} \bar{d}$ are obtained.
\end{abstract}

\section{Introduction}

Since the charmonium-like resonance $X(3872)$ is observed by the Bell Collaboration [1] in 2003, a lot of experiments have emerged to study the exotic states- $X Y Z$ particles from the Belle, BaBar, BESIII, LHCb, CDF, D0, and other Collaborations. Some believe that the traditional convention, that the meson is made up of quark and antiquark as well as baryon is made up of three quarks, is broken. The exotic states were observed in $B$ meson decays, in $e^{+} e^{-}$and $\bar{p} p$ annihilations. In the study of $B$ decays, the phenomenon of $C P$ violation has been studied by experimental collaborations. Many predictions of Standard Model are confirmed and some hints beyond Standard Model are exposed.

Very recently, the D0 Collaboration observed a narrow structure, named $X(5568)$, in the $B_{s}^{0} \pi^{ \pm}$invariant mass spectrum with $5.1 \sigma$ significance [2]. The mass and width measured is $M=5567.8 \pm 2.9_{-1.9}^{+0.9} \mathrm{MeV}$ and $\Gamma=21.9 \pm$ $6.4_{-2.5}^{+5.0} \mathrm{MeV}$, respectively. Its decay mode $B_{s}^{0} \pi^{ \pm}$indicates that $X(5568)$ is consist of four different flavors: $u, d, s, b$. $X(5568)$ must be a $s u \bar{b} \bar{d}$ or $s d \bar{b} \bar{u}$ tetraquark state. The D0 Collaboration suggests that the quantum numbers of

a e-mail: jlping@ @jnu.edu.cn
$X$ (5568) may be $J^{P}=0^{+}$because $B_{s}^{0} \pi^{ \pm}$is produced in $S$-wave. However, the preliminary results of the experimental search of the state by the LHCb Collaboration is negative [3].

The discovery of the exotic state $X(5568)$ stimulated the theoretical interest. Much theoretical work has been done, such as approaches based on QCD sum rules [4-9], quark models [10-12], rescattering effects [14], etc. Agaev et al. studied the state $X(5568)$ within the two-point sum rule method using the diquark-antidiquark interpolating current $[4,15]$ and meson molecule structure [16], their results preferred diquark-antidiquark picture rather than molecule and a nice agreement with experimental data is obtained. QCD sum rule method was also employed by other groups to investigate the state $X(5568)$ as the diquark-antidiquark type scalar and axial-vector tetraquark states [5-9]. In Ref. [10], a tetraquark interpretation of the $X(5568)$ was proposed based on the diquark-antidiquark scheme, the identification is possible when the systematic errors of the model is taken into account. This result is supported by simple quark model estimations $[12,13]$. The hadronic molecule scenarios of the $X(5568)$ is also possible according to the calculation of Ref. [11]. However, there are several theoretical calculations with negative results. Burns and Swanson examined the various interpretations of the state $X(5568)$ and concluded that the threshold, cusp, molecular, and tetraquark models are all unfavored [17]. Guo et al. provided additional arguments using general properties of QCD and obtained the same conclusion [18]. Although the state $X(5568)$ can be reproduced in the coupled channel analysis in Ref. [19], the momentum cutoff used is much larger than the normal one.

Considering the quantum numbers $J^{P}=0^{+}$of the state $X(5568)$, the spin and orbit angular momentum can both be taken as zero. For the meson molecule structure, the possible channels are $B_{s}^{0} \pi, B_{s}^{*} \rho, B^{+} \bar{K}^{0}$, and $B^{*+} \bar{K}^{*}{ }^{0}$. For the diquark-antidiquark structure, the only possible state is $s u \bar{b} \bar{d}$ for $X(5568)^{+}$or $s d \bar{b} \bar{u}$ for $X(5568)^{-}$. In the present work, we compute all these states including the molecule and diquark- 


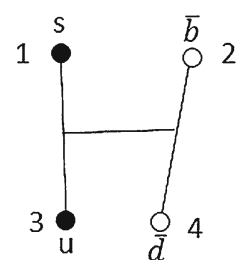

(a)

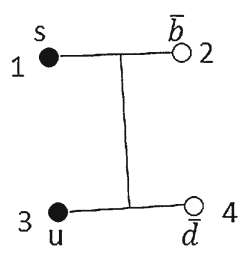

(b) (c)

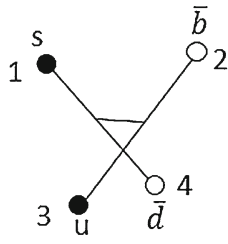

Fig. 1 Structure of the tetraquark $u s \bar{d} \bar{b}$ system. Solid and open circles represent quarks and antiquarks, respectively. a Diquark-antidiquark channel, b direct meson-meson channel: $B_{s}^{0} \pi^{+}$or $B_{s}^{*} \rho$, c exchange meson-meson channel: $B^{+} \bar{K}^{0}$ or $B^{*+} \bar{K}^{*}$

antidiquark structures using the chiral quark model under the flavor $S U$ (3) and $S U$ (4) symmetry, respectively. Besides, we extend our investigation to the new family of the four flavor exotic states $X_{c}$ with $u, d, s, c$ by replacing the $b$ quark with a $c$ quark. We hope that we can find some useful and meaningful information of $X(5568)$ through our systematic calculations.

This article is organized as follows. In Sect. 2, we introduce the Gaussian Expansion Method (GEM) and chiral quark model. In the next section, the numerical calculations with discussions are presented. A short summary is given in the last section.

\section{GEM and chiral quark model}

In the chiral quark model, the mass of the tetraquark state is obtained by solving the Schrödinger equation,

$H \Psi_{M_{I} M_{J}}^{I J}=E^{I J} \Psi_{M_{I} M_{J}}^{I J}$

where $\Psi_{M_{I} M_{J}}^{I J}$ is the wave function of the tetraquark state, which can be constructed as follows. First, we write down the wave functions of two clusters (taking the meson-meson configuration as an example),

$\Psi_{M_{I_{1}} M_{J_{1}}}^{I_{1} J_{1}}(12)=\left[\psi_{l_{1}}\left(\mathbf{r}_{12}\right) \chi_{s_{1}}(12)\right]_{M_{J_{1}}}^{J_{1}} \omega^{c_{1}}(12) \phi_{M_{I_{1}}}^{I_{1}}(12)$,

$\Psi_{M_{I_{2}} M_{J_{2}}}^{I_{2} J_{2}}(34)=\left[\psi_{l_{2}}\left(\mathbf{r}_{34}\right) \chi_{s_{2}}(34)\right]_{M_{J_{2}}}^{J_{2}} \omega^{c_{2}}(34) \phi_{M_{I_{2}}}^{I_{2}}(34)$,

where $\chi_{s}, \omega^{c}, \phi^{I}$ are spin, color and flavor wave functions of the quark-antiquark cluster (the quarks are numbered as 1, 3 and antiquarks 2, 4; see Fig. 1). [ ] denotes the angular momentum coupling. Then the total wave function of the tetraquark state is obtained,

$$
\begin{aligned}
\Psi_{M_{I} M_{J}}^{I J}= & \mathcal{A}\left[\Psi^{I_{1} J_{1}}(1,2) \Psi^{I_{2} J_{2}}(3,4) \psi_{L_{r}}\left(\mathbf{r}_{1234}\right)\right]_{M_{I} M_{J}}^{I J} \\
= & {\left[\left[\psi_{l_{1}}\left(\mathbf{r}_{12}\right) \chi_{s}(12)\right]^{J_{1}}\left[\psi_{l_{2}}\left(\mathbf{r}_{34}\right) \chi_{s}(34)\right]^{J_{2}}\right.} \\
& \left.\times \psi_{L_{r}}\left(\mathbf{r}_{1234}\right)\right]_{M_{J}}^{J}\left[\omega^{c_{1}}(12) \omega^{c_{2}}(34)\right]^{[222]} \\
& \times\left[\phi_{M_{I_{1}}}^{I_{1}}(12) \phi_{M_{I_{2}}}^{I_{2}}(34)\right]_{M_{I}}^{I},
\end{aligned}
$$

where $\psi_{L_{r}}\left(\mathbf{r}_{1234}\right)$ is the relative wave function between two clusters with the relative orbit angular momentum $L_{r} . \mathcal{A}$ is the antisymmetrization operator. If all quarks (antiquarks) are taken as identical particles, we have

$\mathcal{A}=\frac{1}{2}\left(1-P_{13}-P_{24}+P_{13} P_{24}\right)$.

In GEM, the orbital wave function is written as the product of radial one and spherical harmonics, and the radial part of the wave function is expanded by Gaussians,

$$
\begin{gathered}
\psi_{l m}(\mathbf{r})=\sum_{n=1}^{n_{\max }} c_{n} \psi_{n l m}^{G}(\mathbf{r}), \\
\psi_{n l m}^{G}(\mathbf{r})=N_{n l} r^{l} e^{-v_{n} r^{2}} Y_{l}^{m}(\hat{\mathbf{r}}) .
\end{gathered}
$$

The Gaussian size parameters are taken as the following geometric progression numbers:

$v_{n}=\frac{1}{r_{n}^{2}}, \quad r_{n}=r_{1} a^{n-1}, \quad a=\left(\frac{r_{n_{\max }}}{r_{1}}\right)^{\frac{1}{n_{\max }-1}}$.

Noting that the Gaussians are not orthogonal, the RayleighRitz variational principle for solving the Schrödinger equation leads to a generalized eigenvalue problem,

$$
\begin{aligned}
& \sum_{n^{\prime} \alpha^{\prime}}\left(H_{n \alpha, n^{\prime} \alpha^{\prime}}^{I J}-E^{I J} N_{n \alpha, n^{\prime} \alpha^{\prime}}^{I J}\right) C_{n^{\prime} \alpha^{\prime}}^{I J}=0, \\
& H_{n \alpha, n^{\prime} \alpha^{\prime}}^{I J}=\left\langle\Phi_{n \alpha}^{I M_{I} J M_{J}}|H| \Phi_{n^{\prime} \alpha^{\prime}}^{I M_{I} J M_{J}}\right\rangle, \\
& N_{n \alpha, n^{\prime} \alpha^{\prime}}^{I J}=\left\langle\Phi_{n \alpha}^{I M_{I} J M_{J}} \mid \Phi_{n^{\prime} \alpha^{\prime}}^{I M_{I} J M_{J}}\right\rangle,
\end{aligned}
$$

where $\alpha$ denotes channels.

The Hamiltonian of the chiral quark model includes three parts, the rest masses of quarks, the kinetic energy and the potential energy. The potential energy is composed of color confinement, one-gluon exchange, and one-Goldstone boson exchange. The detailed form for the tetraquark states is shown below [20],

$$
\begin{aligned}
H= & \sum_{i=1}^{4} m_{i}+\frac{p_{12}^{2}}{2 \mu_{12}}+\frac{p_{34}^{2}}{2 \mu_{34}}+\frac{p_{1234}^{2}}{2 \mu_{1234}} \\
& +\sum_{i<j=1}^{4}\left(V_{i j}^{G}+V_{i j}^{C}+\sum_{\chi=\pi, K, \eta} V_{i j}^{\chi}+V_{i j}^{\sigma}\right),
\end{aligned}
$$




$$
\begin{aligned}
V_{i j}^{G}= & \frac{\alpha_{s}}{4} \lambda_{i}^{c} \cdot \lambda_{j}^{c}\left[\frac{1}{r_{i j}}-\frac{2 \pi}{3 m_{i} m_{j}} \sigma_{i} \cdot \boldsymbol{\sigma}_{j} \delta\left(\boldsymbol{r}_{i j}\right)\right], \\
& \delta\left(\boldsymbol{r}_{i j}\right)=\frac{e^{-r_{i j} / r_{0}\left(\mu_{i j}\right)}}{4 \pi r_{i j} r_{0}^{2}\left(\mu_{i j}\right)}, \\
V_{i j}^{C}= & \left(-a_{c} r_{i j}^{2}-\Delta\right) \lambda_{i}^{c} \cdot \lambda_{j}^{c} \\
V_{i j}^{\pi}= & \frac{g_{c h}^{2}}{4 \pi} \frac{m_{\pi}^{2}}{12 m_{i} m_{j}} \frac{\Lambda_{\pi}^{2}}{\Lambda_{\pi}^{2}-m_{\pi}^{2}} m_{\pi} v_{i j}^{\pi} \sum_{a=1}^{3} \lambda_{i}^{a} \lambda_{j}^{a}, \\
V_{i j}^{K}= & \frac{g_{c h}^{2}}{4 \pi} \frac{m_{K}^{2}}{12 m_{i} m_{j}} \frac{\Lambda_{K}^{2}}{\Lambda_{K}^{2}-m_{K}^{2}} m_{K} v_{i j}^{K} \sum_{a=4}^{7} \lambda_{i}^{a} \lambda_{j}^{a}, \\
V_{i j}^{\eta}= & \frac{g_{c h}^{2}}{4 \pi} \frac{m_{\eta}^{2}}{12 m_{i} m_{j}} \frac{\Lambda_{\eta}^{2}}{\Lambda_{\eta}^{2}-m_{\eta}^{2}} m_{\eta} v_{i j}^{\eta} \\
& \times\left[\lambda_{i}^{8} \lambda_{j}^{8} \cos \theta_{P}-\lambda_{i}^{0} \lambda_{j}^{0} \sin \theta_{P}\right], \\
V_{i j}^{\sigma}= & -\frac{g_{c h}^{2}}{4 \pi} \frac{\Lambda_{\sigma}^{2}}{\Lambda_{\sigma}^{2}-m_{\sigma}^{2}} m_{\sigma}\left[Y\left(m_{\sigma} r_{i j}\right)-\frac{\Lambda_{\sigma}}{m_{\sigma}} Y\left(\Lambda_{\sigma} r_{i j}\right)\right] \\
v_{i j}^{\chi}= & {\left[Y\left(m_{\chi} r_{i j}\right)-\frac{\Lambda_{\chi}^{3}}{m_{\chi}^{3}} Y\left(\Lambda_{\chi} r_{i j}\right)\right] \sigma_{i} \cdot \sigma_{j}, } \\
& Y(x)=e^{-x} / x,
\end{aligned}
$$

where $m_{i}$ is the mass of quarks and antiquarks, and $\mu_{i j}$ is their reduced mass, $r_{0}\left(\mu_{i j}\right)=\hat{r}_{0} / \mu_{i j}, \sigma$ are the $S U$ (2) Pauli matrices, $\lambda, \lambda^{c}$ are $S U$ (3) flavor, color Gell-Mann matrices, $g_{c h}^{2} / 4 \pi$ is the chiral coupling constant, determined from the $\pi$-nucleon coupling constant. $\alpha_{s}$ is the effective scaledependent running quark-gluon coupling constant [20],

$\alpha_{s}\left(\mu_{i j}\right)=\frac{\alpha_{0}}{\ln \left[\left(\mu_{i j}^{2}+\mu_{0}^{2}\right) / \Lambda_{0}^{2}\right]}$.

All model parameters are determined by fitting the meson spectrum and shown in Table 1. The calculated masses of the mesons involved in the present work are shown in Table 2.

\section{Numerical results}

In the present calculation, two structures of four-quark states, diquark-antidiquark and meson-meson, are investigated. In each structure, all possible states are considered. For diquark-antidiquark structure, two color configurations, color antitriplet-triplet $(\overline{3} \times 3)$ and sextet-antisextet $(6 \times \overline{6})$ are taken into account. For meson-meson structure, two color configurations, color singlet-singlet $(1 \times 1)$ and octet-octet $(8 \times 8)$ are employed.

The calculation with the ordinary flavor symmetry, $S U(3)$ is first performed, i.e., we have no Goldstone boson exchanges between $u, d, s$, and $b$ quark. In this case, the
Table 1 Quark model parameters

\begin{tabular}{lll}
\hline Quark masses & $m_{u}=m_{d}(\mathrm{MeV})$ & 313 \\
& $m_{s}(\mathrm{MeV})$ & 536 \\
& $m_{c}(\mathrm{MeV})$ & 1728 \\
& $m_{b}(\mathrm{MeV})$ & 5112 \\
Goldstone bosons & $m_{\pi}\left(\mathrm{fm}^{-1}\right)$ & 0.70 \\
& $m_{\sigma}\left(\mathrm{fm}^{-1}\right)$ & 3.42 \\
& $m_{\eta}\left(\mathrm{fm}^{-1}\right)$ & 2.77 \\
& $m_{K}\left(\mathrm{fm}^{-1}\right)$ & 2.51 \\
& $\Lambda_{\pi}=\Lambda_{\sigma}\left(\mathrm{fm}^{-1}\right)$ & 4.2 \\
& $\Lambda_{\eta}=\Lambda_{K}\left(\mathrm{fm}^{-1}\right)$ & 5.2 \\
$g_{c h}^{2} /(4 \pi)$ & 0.54 \\
& $\theta_{p}\left({ }^{\circ}\right)$ & -15 \\
Confinement & $a_{c}(\mathrm{MeV})$ & 101 \\
& $\Delta(\mathrm{MeV})$ & -78.3 \\
OGE & $\alpha_{0}$ & 3.67 \\
& $\Lambda_{0}\left(\mathrm{fm}{ }^{-1}\right)$ & 0.033 \\
& $\mu_{0}(\mathrm{MeV})$ & 36.976 \\
$\hat{r}_{0}(\mathrm{MeV})$ & 28.17 \\
\hline
\end{tabular}

Table 2 Meson spectrum (unit: MeV)

\begin{tabular}{lrc}
\hline Meson & Energy & Experimental value \\
\hline$B_{s}^{0}$ & 5368 & 5366 \\
$\pi$ & 139 & 139 \\
$B_{s}^{*}$ & 5410 & 5415 \\
$\rho$ & 772 & 770 \\
$B^{+}$ & 5281 & 5279 \\
$\bar{K}^{0}$ & 494 & 497 \\
$B^{*+}$ & 5320 & 5325 \\
$\bar{K}^{*}$ & 914 & 892 \\
$D_{s}^{-}$ & 1953 & 1968 \\
$\bar{D}^{0}$ & 1862 & 1864 \\
\hline
\end{tabular}

antisymmetrization operator used is

$\mathcal{A}=\sqrt{\frac{1}{2}}\left(1-P_{13}\right)$

The results in this case are listed in Table 3.

From Table 3, we can see that the two configurations of the diquark-antidiquark structure, $\overline{3} \times 3$ and $6 \times \overline{6}$, have similar energies, and the coupling between the two configurations is rather strong. Nevertheless, the energy for the diquarkantidiquark structure is too large to be a natural candidate of the state $X(5568)$ in our calculation, although it could be a resonance because of its color structure. With regard to meson-meson structure, the calculated energies approach to the theoretical thresholds in all case. Thus, no molecular 
Table 3 The energies of tetraquark system $s u \bar{d} \bar{b}$ with flavor $S U(3)$ symmetry. $E_{\text {th }}^{\text {theo }}$ is the theoretical threshold value and $E_{\mathrm{th}}^{\exp }$ represents the experimental threshold value (unit: $\mathrm{MeV}$ )

\begin{tabular}{|c|c|c|c|c|c|}
\hline$q q-\bar{q} \bar{q}$ & $E_{\overline{3} \otimes 3}$ & $E_{6 \otimes \overline{6}}$ & $E_{c c}$ & & \\
\hline$s u \bar{d} \bar{b}$ & 6406.0 & 6473.6 & 6360.0 & & \\
\hline$q \bar{q}-q \bar{q}$ & $E_{1 \otimes 1}$ & $E_{8 \otimes 8}$ & $E_{c c}$ & $E_{\mathrm{th}}^{\text {theo }}$ & $E_{\mathrm{th}}^{\exp }$ \\
\hline$B_{s}^{0} \pi$ & 5509.5 & 6443.5 & 5509.5 & 5507 & 5505 \\
\hline$B_{s}^{*} \rho$ & 6185.5 & 6345.3 & 6185.5 & 6182 & 6185 \\
\hline$B_{s}^{0} \pi-B_{s}^{*} \rho$ & 5509.5 & 6324.3 & 5509.5 & 5507 & 5505 \\
\hline$B^{+} \bar{K}^{0}$ & 5776.8 & 6519.5 & 5776.8 & 5774 & 5776 \\
\hline$B^{*+} \bar{K}^{*}{ }^{0}$ & 6235.2 & 6403.9 & 6235.2 & 6233 & 6217 \\
\hline$B^{+} \bar{K}^{0}-B^{*+} \bar{K}^{* 0}$ & 5776.8 & 6376.9 & 5776.8 & 5774 & 5776 \\
\hline
\end{tabular}

Table 4 The energies of tetraquark system $s u \bar{d} \bar{b}$ with flavor $S U(4)$ symmetry. $E_{\text {th }}^{\text {theo }}$ is the theoretical threshold value and $E_{\mathrm{th}}^{\exp }$ represents the experimental threshold value. (unit: $\mathrm{MeV}$ )

\begin{tabular}{llllll}
\hline$q q-\bar{q} \bar{q}$ & $E_{\overline{3} \otimes 3}$ & $E_{6 \otimes \overline{6}}$ & $E_{c c}$ & & \\
\hline$s u \bar{d} \bar{b}$ & 6397.6 & 6466.4 & 6351.0 & & \\
\hline$q \bar{q}-q \bar{q}$ & $E_{1 \otimes 1}$ & $E_{8 \otimes 8}$ & $E_{c c}$ & $E_{\text {th }}^{\text {theo }}$ & $E_{\text {th }}^{\text {exp }}$ \\
\hline$B_{s}^{0} \pi$ & 5522.0 & 6431.1 & 5522.0 & 5518 & 5505 \\
$B_{s}^{*} \rho$ & 6282.7 & 6324.3 & 6182.5 & 6177 & 6185 \\
$B_{s}^{0} \pi-B_{s}^{*} \rho$ & 5522.0 & 6306.1 & 5521.0 & 5518 & 5505 \\
$B^{+} \bar{K}^{0}$ & 5717.6 & 6440.1 & 5717.6 & 5715 & 5776 \\
$B^{*+} \bar{K}^{*}{ }^{+}$ & 6204.6 & 6277.2 & 6204.5 & 6202 & 6217 \\
$B^{+} \bar{K}^{0}-B^{*+} \bar{K}^{* 0}$ & 5717.6 & 6245.1 & 5717.0 & 5715 & 5776 \\
\hline
\end{tabular}

structure formed in our model calculation. In our calculations, the color singlet-singlet configurations always have the lower energies than that of color octet-octet ones. The coupling between the two configurations is very small. The reason for the small coupling can be understood as follows. The effect of the $K$-meson exchange is too weak to push the energy of color singlet-singlet below the threshold, so the two colorless clusters tend to stay apart. While two colorful clusters prefer to stay close, the overlap between the two configurations is small, so the coupling from the exchange term of the $K$-meson is small.

In the study of $N^{*}$ with hidden charm, the flavor $S U$ (4) symmetry plays an important role [21,22]. To see the effect of flavor $S U$ (4) symmetry, we extend our calculation from flavor $S U(3)$ symmetry to $S U(4)$. In this case, the Goldstone boson exchanges including $\pi, K, \eta, B, B_{s}, \eta_{b}$, totally 15 pseudo-scalar mesons. For scalar mesons, we use effective $\sigma$-meson exchange instead of 16 scalar mesons [23]. The mass of effective $\sigma$-meson takes the average of the quark pairs, $u \bar{u}, d \bar{d}, s \bar{s}$ and $b \bar{b}$, due to its nature of flavor singlet of $S U(4)$. In this work, we take different $m_{\sigma}^{\text {eff }}$ between two different quarks. For example, for the $u$ and $s$ quark, $m_{\sigma}^{\text {eff }}=\left(2 m_{u}+2 m_{s}\right) / 2=849 \mathrm{MeV}$, or $4.3 \mathrm{fm}^{-1}$, the corresponding cutoff takes the value $6.3 \mathrm{fm}^{-1}$. The results with flavor $S U$ (4) symmetry are shown in Table 4. From the table, we can see that the results are almost the same as that of $S U$ (3). That is, no molecular state formed and the energy for the diquark-antidiquark structure is too large to be a candidate of the state $X$ (5568). So in our quark model approach, the $X(5568)$ cannot be explained as molecule or diquarkantidiquark state under the constraint that the model describes the meson spectrum well. Because the state $X$ (5568) involves pseudo-scalar mesons, we attribute the negative results to the Goldstone nature of the light pseudo-scalar mesons, which have extraordinary small masses. Our results are consistent with the analysis of Burns and Swanson [17]. They explored a lot of possible explanations of the $X(5568)$ signal such as a tetraquark, a hadronic molecule or a threshold effect and found that none of them can be a candidate of the observed state. Guo provided addition arguments to the negative results by the way of general properties of QCD [18]. Moreover, the preliminary results of $\mathrm{LHCb}$ do not confirm the state $X(5568)$.

Our results are different from some results of the previous work. In the QCD sum rule approach, Refs. [4,5,7,9] obtain consistent results with experimental data by choosing appropriate Borel and threshold parameters for the diquarkantidiquark structure. However, Zanetti et al. [6] argued that a larger threshold parameter should be used to ensure the convergence of OPE, then they get a much larger mass of the 
Table 5 The energies of tetraquark system $s u \bar{c} \bar{d}$. $E_{\text {th }}^{\text {theo }}$ is the theoretical threshold value and $E_{\mathrm{th}}^{\exp }$ represents the experimental threshold value. (unit: $\mathrm{MeV}$ )

\begin{tabular}{llllll}
\hline$S U(3)$ & & & & \\
\hline$q q-\bar{q} \bar{q}$ & $E_{\overline{3} \otimes 3}$ & $E_{6 \otimes \overline{6}}$ & $E_{c c}$ & & \\
\hline$s u \bar{c} \bar{d}$ & 3059.0 & 3073.9 & 2983 & & \\
\hline$q \bar{q}-q \bar{q}$ & $E_{1 \otimes 1}$ & $E_{8 \otimes 8}$ & $E_{c c}$ & $E_{\text {th }}^{\text {theo }}$ & $E_{\text {th }}^{\exp }$ \\
\hline$D_{s}^{-} \pi$ & 2095.1 & 3080.6 & 2095.1 & 2092 & 2107 \\
$\bar{K}^{0} \bar{D}^{0}$ & 2358.7 & 3133.8 & 2358.7 & 2355 & 2361 \\
\hline$S U(4)$ & & & & & \\
\hline$q q-\bar{q} \bar{q}$ & $E_{\overline{3} \otimes 3}$ & $E_{6 \otimes \overline{6}}$ & $E_{c c}$ & & \\
$s u \bar{c} \bar{d}$ & 3023.4 & 3073.9 & 2943 & & \\
$q \bar{q}-q \bar{q}$ & $E_{1 \otimes 1}$ & $E_{8 \otimes 8}$ & $E_{c c}$ & $E_{\text {th }}^{\text {theo }}$ & $E_{\text {th }}^{\exp }$ \\
\hline$D_{s}^{-} \pi$ & 2088.7 & 3043.6 & 2088.6 & 2085 & 2107 \\
$\bar{K}^{0} \bar{D}^{0}$ & 2279.2 & 3073.2 & 2279.2 & 2276 & 2361 \\
\hline
\end{tabular}

state, $6390 \mathrm{MeV}$, which is very close to our results, 6351$6360 \mathrm{MeV}$.

In the quark model approach, several calculations obtain the mass of the state in the region of the experimental data by using a simplified Hamiltonian which includes only the mass term and chromomagnetic term. Wang and Zhu get a mass of the scalar $s u \bar{d} \bar{b}$ around $5708 \mathrm{MeV}$, which is not far from the experimental data. As pointed out by Burns and Swanson [17], the not so large mass comes from the lighter diquark masses that are used. Much lower masses of the tetraquark state with $J=0,1$ are obtained by Liu et al. [12]. Comparing the parameters of quark model, the low masses of the states are attributed to the small masses of quarks used; e.g., the mass of $b$-quark is $4630 \mathrm{MeV}$, which is much smaller than the value used here, $5112 \mathrm{MeV}$. Burns and Swanson point out that the smaller quark masses will lead to an under-estimate of the heavy baryon masses [17]. In Ref. [13], Stancu obtained a mass of $5530 \mathrm{MeV}$ for the scalar tetraquark $s u \bar{d} \bar{b}$, where the chromomagnetic interaction is simplified as $\sum_{i j} C_{i j} \sigma_{i} \cdot \sigma_{j} \lambda_{i} \cdot \lambda_{j}$, and the coefficients $C_{i j}$ are extracted from a global fit to meson and baryon ground states. However, a large spin splitting of $-552 \mathrm{MeV}$ will be induced according to the analysis of Burns and Swanson [17].

The calculation is also extended to the system composed of four different quarks: $s, u, \bar{c}, \bar{d}$, replacing the mass of heavy quark $\bar{b}$ by $\bar{c}$. The results are shown in Table 5 .

From Table 5, we can obtain the same conclusion as that of the $s u \bar{c} \bar{d}$ system. The masses of the system in the diquarkantidiquark structure are too large and in the meson-meson molecular structure approach the thresholds. Our calculation disfavor the existence of an exotic suc $\bar{c} \bar{d}$ state. The results are consistent with the general expectation that the heavier the system is, the stronger the states be bound.

\section{Summary}

In this paper we have studied the new exotic resonance state $X(5568)$ with the quantum numbers, $J^{P}=0^{+}$, which was observed recently by the D0 Collaboration utilizing the collected data of $p \bar{p}$ collisions. The constituent chiral quark model, which describes the light and heavy meson spectra well, is employed in the calculation. Two structures: diquark-antidiquark and meson-meson, with flavor symmetries, $S U(3)$ and $S U(4)$, are investigated. We find that the masses of $u s \bar{b} \bar{d}$ with diquark-antidiquark structure are too high to be candidates of the state $X(5568)$ and no molecular structure can be formed. The calculation is extended to the $u s \bar{c} \bar{d}$ system, and the same conclusion is obtained.

Because of the quark contents of the system, the pseudoscalar mesons are involved. The extraordinary small masses of these Goldstone bosons disfavor the existence of the exotic. Our results agree with the analysis of Burns and Swanson. The recent preliminary results of LHCb Collaboration do not confirm the state $X(5568)$, so more experimental and theoretical work are needed to clarify the situation.

Acknowledgments The work is supported partly by the National Natural Science Foundation of China under Grant Nos. 11175088 and 11535005

Open Access This article is distributed under the terms of the Creative Commons Attribution 4.0 International License (http://creativecomm ons.org/licenses/by/4.0/), which permits unrestricted use, distribution, and reproduction in any medium, provided you give appropriate credit to the original author(s) and the source, provide a link to the Creative Commons license, and indicate if changes were made. Funded by SCOAP ${ }^{3}$.

\section{References}

1. S.-K. Choi et al., Belle collaboration. Phys. Rev. Lett. 91, 262001 (2003)

2. V. M. Abazov et al. [D0 Collaboration]. arXiv:1602.07588 [hep-ex]

3. The LHCb Collaboration [LHCb Collaboration], LHCbCONF2016-004, CERN-LHCb-CONF-2016-004

4. S. S. Agaev, K. Azizi, H. Sundu. arXiv:1602.08642 [hep-ph]

5. Z. G. Wang. arXiv:1602.08711 [hep-ph], arXiv:1603.02498 [hep$\mathrm{ph}]$

6. C. M. Zanetti, M. Nielsen, K. P. Khemchandani. arXiv:1602.09041 [hep-ph]

7. W. Chen, H. X. Chen, X. Liu, T. G. Steele, S. L. Zhu. arXiv: 1602.08916 [hep-ph]

8. J. M. Dias, K. P. Khemchandani, A. M. Torres et al. arXiv:1603.02249 [hep-ph]

9. L. Tang, C. F. Qiao. arXiv:1603.04761 [hep-ph]

10. W. Wang, R. L. Zhu. arXiv:1602.08806 [hep-ph]

11. C. J. Xiao, D. Y. Chen, arXiv:1603.002282 [hep-ph]

12. Y. R. Liu, X. Liu, S. L. Zhu. arXiv:1603.01131 [hep-ph]

13. F. Stancu. arXiv: 1603.03322 [hep-ph]

14. X. H. Liu, G. Li. arXiv:1603.00708 [hep-ph]

15. S. S. Agaev, K. Azizi, H. Sundu. arXiv:1603.00290 [hep-ph]

16. S. S. Agaev, K. Azizi, H. Sundu. arXiv:1603.02708 [hep-ph] 
17. T. J. Burns, E. S. Swanson. arXiv:1603.04366 [hep-ph]

18. F. K. Guo, U. G. Meissner, B. S. Zou. arXiv:1603.06316 [hep-ph]

19. M. Albaladejo, J. Nieves, E. Oset, Z. F. Sun, X. Liu. arXiv:1603.09230 [hep-ph]

20. A. Valcarce, H. Garcilazo, F. Fernandez, P. Gonzalez, Rep. Prog. Phys. 68, 965 (2005) (references therein)
21. J.J. Wu, R. Molina, E. Oset, B.S. Zou, Phys. Rev. Lett. 105, 232001 (2010)

22. J.J. Wu, R. Molina, E. Oset, B.S. Zou, Phys. Rev. C 84, 015202 (2011)

23. H. Garcilazo, T. Fernández-Caramés, A. Valcarce, Phys. Rev. C. 75, 034002 (2007) 